University of Nebraska - Lincoln

DigitalCommons@University of Nebraska - Lincoln

$8-2000$

\title{
Nutrient absorption and utilization by wing and flight muscle morphs of the cricket Gryllus firmus: Implications for the trade-off between flight capability and early reproduction
}

Anthony J. Zera

University of Nebraska - Lincoln, azera1@unl.edu

Tammy Brink

University of Nebraska - Lincoln

Follow this and additional works at: https://digitalcommons.unl.edu/bioscizera

Part of the Microbiology Commons

Zera, Anthony J. and Brink, Tammy, "Nutrient absorption and utilization by wing and flight muscle morphs of the cricket Gryllus firmus: Implications for the trade-off between flight capability and early reproduction" (2000). Anthony Zera Publications. 36.

https://digitalcommons.unl.edu/bioscizera/36

This Article is brought to you for free and open access by the Papers in the Biological Sciences at DigitalCommons@University of Nebraska - Lincoln. It has been accepted for inclusion in Anthony Zera Publications by an authorized administrator of DigitalCommons@University of Nebraska - Lincoln. 
Published in Journal of Insect Physiology 46:8 (August 2000), pp. 1207-1218; doi 10.1016/S0022-1910(00)00041-X

Copyright (C) 2000 Elsevier Science Ltd. Used by permission. http://www.sciencedirect.com/science/journal/00221910

Submitted June 21, 1999; accepted January 14, 2000; published online May 15, 2000.

\title{
Nutrient absorption and utilization by wing and flight muscle morphs of the cricket Gryllus firmus: Implications for the trade-off between flight capability and early reproduction
}

\author{
Anthony J. Zera and Tammy Brink \\ School of Biological Sciences, University of Nebraska-Lincoln, NE 68588, USA \\ Corresponding author - A. J. Zera, tel 402 472-2768, fax 402 472-2083, email azera@unlserve.unl.edu
}

\begin{abstract}
Absorption efficiency (AD, approximate digestibility, assimilation efficiency) of various macronutrients and conversion of absorbed nutrients to biomass (ECD) were compared among the two types of flightless morph and the flight-capable morph of the cricket, Gryllus firmus. No biologically significant phenotypic or genetic difference in AD for carbohydrate, protein or lipid was observed among morphs fed either a high-nutrient (100\%) or a low-nutrient (25\%) diet. Thus, previously-documented differences among adult morphs in carbohydrate and lipid content must be caused by processes other than variation in nutrient absorption by morphs during adulthood. Relative absorption efficiency of total dry mass of food by morphs of G. firmus appears to be a valid indicator of relative AD of total calories. Morphs did not differ phenotypically or genetically in the excretion of end products of nitrogen metabolism (uric acid, hypoxanthine plus xanthine) on either the high nutrient or the low nutrient diet. Nutritional indices corrected for excreted nitrogenous metabolites were very similar to uncorrected indices, and the pattern of variation among the morphs was the same for corrected or uncorrected values. Each of the two types of flightless morph converted a greater proportion of absorbed nutrients into body mass, mainly ovaries, and allocated a smaller proportion of assimilated nutrients to respiration than did the flight-capable morph. Moreover, the trade-off between respiration and early reproduction was substantially magnified on the low nutrient diet. These results extend previous findings of a trade-off between flight capability and early reproduction in wing-polymorphic Gryllus species (1) to diets of very different nutrient quantity, and (2) to flightlessness arising from different causes: blockage of flight muscle development in juveniles vs histolysis of fully-developed flight muscles in adults.
\end{abstract}

Keywords: wing polymorphism, nutritional indices, absorption, assimilation, AD, ECD, trade-off, crickets, Gryllus

\section{Introduction}

Wing polymorphism is a phylogenetically-widespread and ecologically important feature of insects (Harrison, 1980; Roff, 1986; Dingle, 1996; Zera and Denno, 1997). The polymorphism involves discontinuous variation in a wide variety of traits involved in dispersal and reproduction. One morph has fully developed (long) wings and flight muscles and is capable of dispersal [denoted as "LW(f)"]. Another morph never fully develops wings and flight muscles and is obligately flightless [denoted "SW"]. Finally, in many species, a second flightless morph is derived from long-winged adults by histolysis of the fully-developed flight muscles [denoted "LW(h)";
Zera et al., 1997]. Importantly, in each of the flightless morphs, ovarian growth begins earlier than in the flightcapable, long-winged morph. That is, flight capability is negatively associated with (i.e. trades off with) reproductive output (Roff, 1986; Dingle, 1996; Zera and Denno, 1997).

Wing polymorphism has been used extensively to investigate ecological and evolutionary aspects of dispersal, physiology and development (Harrison, 1980; Hardie and Lees, 1985; Roff, 1986; Dingle, 1996; Zeraand Denno, 1997; Zera et al., 1998; Nijhout, 1999). One major line of physiological research has focused on energetic aspects of the trade-off between ovarian growth and flight capability in crickets of the genus Gryllus (Mole 
and Zera, 1993; Zera and Mole, 1994; Zera and Denno, 1997; Zera et al., 1998). The most important finding of these studies is that flightless females, in each of three Gryllus species, converted a greater proportion of absorbed nutrients into biomass (mainly ovaries) during the first 1-2 weeks of adulthood. This increased degree of nutrient conversion (increased ECD; see Section 2 for definitions) by flightless females results from decreased energetic (respiratory) costs associated with that morph. These decreased costs have not been definitively identified, but they most likely result from reduced flight muscle maintenance, reduced lipid (flight fuel) biosynthesis, or a reduction in the general activity of flightless individuals (Zera et al., 1998).

In contrast to the consistently elevated ECD of flightless females of three Gryllus species, nutrient absorption (AD, absorption efficiency, approximate digestibility; assimilation efficiency; Waldbauer, 1968; Woodring et al., 1979; van Loon, 1989) differed only modestly or not at all between morphs of these species (Mole and Zera, 1993; Zera and Mole, 1994; Zera et al., 1998; see Section 4). Hence, increased conversion of absorbed nutrients was viewed as being more important than increased absorption of nutrients in providing the material for increased egg production of flightless females during early adulthood (Zera and Denno, 1997; Zera et al., 1998).

As had been done in many nutritional index studies in insects (Waldbauer, 1968; Slansky and Scriber, 1985; van Loon, 1989), nutritional indices were estimated in various Gryllus species by quantifying the dry masses of food consumed and material excreted, coupled with the dry mass gain of individual crickets. Measurement of absorption by this method has several well-known shortcomings (Waldbauer, 1968; Slansky and Scriber, 1985; van Loon, 1989). For example, excreted material consists of end products of nitrogen metabolism (e.g. uric acid), as well as unabsorbed food. The failure to determine the proportion of excreta that results from metabolic end products, as opposed to unabsorbed food, introduces errors into estimates of $\mathrm{AD}$ and other indices based on absorption (e.g. ECD). Furthermore, AD, calculated from the dry masses of food consumed and material excreted, provides no information on assimilation of individual nutrients such as carbohydrate or lipid. The failure to take into account differences between morphs in the absorption of various nutrients could lead to erroneous conclusions about the energetic causes of morphspecific adaptations (see Section 4).

The main purpose of the present study was to obtain information on absorption of various macronutrients and the excretion of end products of nitrogen metabolism by wing and flight muscle morphs of Gryllus. We were especially interested in determining whether wing morphs differ in these features and the effects of any differences on estimates of nutritional indices. Further- more, we extended our previous nutritional index studies by separately estimating $\mathrm{AD}$ and ECD for the two types of flightless morphs [LW(h) and SW], as well as the flight capable LW(f). Like most earlier physiological and ecological studies of wing polymorphism, our previous studies compared nutritional indices of morphs that differ in wing length (LW and SW females) without separating LW individuals into their two component flight-muscle morphs (Zera and Denno, 1997). Recent physiological studies indicate that the failure to separate the two types of LW morphs can result in substantial errors in estimates of both fitness and energetic costs of flightlessness (Zera et al., 1997).

\section{Materials and methods}

\subsection{Stock descriptions, sampling protocol and rearing conditions}

Gryllus firmus, the sand cricket, occurs in the southeastern United States (Veazy et al., 1976) as three wing and flight-muscle morphs [LW(f), LW(h) and SW; see Section 1 and Zera et al. (1997)]. G. firmus used in the present study were taken from two types of lines that are part of an ongoing artificial selection study: (1) unselected control lines, and (2) lines selected for either the LW or SW morph (see Zera and Huang, 1999). Artificial selection is being conducted in three independent trials (blocks), and the numbers following the various lines indicate the selection trial to which the lines belong. Here, "CON" refers to a control (unselected) line from which both LW and SW individuals were sampled. "SEL" represents a pair of lines from the same block, in which $\mathrm{LW}(\mathrm{f})$ and $\mathrm{LW}(\mathrm{h})$ individuals were taken from the LWselected line, and SW individuals were taken from the SW-selected line. Sampling morphs from control and selected lines allowed us to determine whether morphspecific differences in nutrient assimilation exist in the base population (CON lines) and whether genetic variation exists for nutrient assimilation [i.e. variation between LW-selected and SW-selected morphs from a pair of selected (SEL) lines]. Lines were sampled during the 5 th-6th generations of selection. During these generations, the frequency of the LW morph [combined LW(f) and $L W(h)]$ ranged from 0.71 to 0.82 in the $L W$-selected lines, and $0.64-0.52$ in the control lines. The frequency of the SW-morph ranged from 0.64 to 0.73 in the SWselected lines. During the last stadium, male and female crickets were separated and checked every $24 \mathrm{~h}$ for newly molted adults. Adults were then raised on a high nutrient $(100 \%)$ or low nutrient $(25 \%)$ diet, which are described in more detail below. Only females were examined in the present study. All crickets were reared under a $16 \mathrm{~h}$ light: $8 \mathrm{~h}$ dark photoregime at $28^{\circ} \mathrm{C}$. 


\subsection{Extractions of macronutrients and metabolites}

Protein and carbohydrate were extracted together from excretory material as follows: $25 \mathrm{mg}$ of freeze-dried excreta was powdered and mixed with $1 \mathrm{ml}$ of $0.1 \mathrm{M}$ $\mathrm{NaOH}$. Tubes were rotated at $8^{\circ} \mathrm{C}$ for $24 \mathrm{~h}$ and samples were then centrifuged at $2000 \mathrm{~g}$ for $5 \mathrm{~min}$. The supernatant was removed and the pellet was resuspended in $500 \mu \mathrm{l} 0.1 \mathrm{M} \mathrm{NaOH}$, re-centrifuged as described above, and the supernatant was combined with the first supernatant. A background study showed that this procedure removed greater than $95 \%$ of the soluble protein and carbohydrate from the excreta. The background study also study showed that there was no degradation of protein during the $24 \mathrm{~h}$ extraction period. However, there was some reduction in carbohydrate concentration presumably due to degradation. To compensate for this loss in carbohydrate, the percent loss of glucose (1 $\mathrm{mg} / \mathrm{ml}$ ) after $24 \mathrm{~h}$ incubation in $0.1 \mathrm{M} \mathrm{NaOH}$ was determined and used to adjust carbohydrate estimates in the excreta. Total lipid was extracted using the method described in Zera et al. (1994). Twenty-five milligrams of powdered excreta were mixed for $24 \mathrm{~h}$ with $3 \mathrm{ml}$ of chloroform/methanol (2:1 v/v), containing $0.05 \%$ butylated hydroxytoluene. The mixture was centrifuged, the supernatant was removed, and $1 \mathrm{ml}$ of chloroform/ methanol was added to the pellet. The pellet was resuspended, centrifuged and the supernatant was removed and combined with the first supernatant. The chloroform/methanol extracts were washed with aqueous $\mathrm{KCl}$ as described in Zera et al. (1994). This procedure removed $>90 \%$ of lipid from the excreta.

Uric acid, hypoxanthine and xanthine were extracted from excretory products of crickets fed the $100 \%$ diet using the method of Bhattacharya and Waldbauer (1969). One milliliter $0.6 \%$ lithium carbonate was added in each of four consecutive 1-h extractions of excreta followed by a $500 \mu \mathrm{l}$ wash. Metabolites in the excreta of crickets fed the $25 \%$ diet were obtained with three 1 -h extractions and a $500 \mu \mathrm{l}$ wash. A preliminary study showed that these procedures extracted greater than $97 \%$ of the uric acid and xanthine+hypoxanthine from the samples.

\subsection{Assays of macronutrients and excretory products}

Soluble protein was measured using the BCA protein assay (Stoschek, 1990) using bovine serum albumin as a standard. The same extracts used in the BCA protein assay also were used to measure soluble carbohydrate by the anthrone assay (Van Handel, 1985) using glucose as a standard. Total lipid was measured by the vanillin assay using oleic acid as the standard (Van Handel, 1985).

Uric acid was quantified using the spectrophotometric assay of Bhattacharya and Waldbauer (1969) with the changes denoted below. In this assay, uricase converts uric acid into allantoin which is quantified by measuring the change in absorbance at $292 \mathrm{~nm}$. The total assay volume was $2500 \mu 1$. 2400-2420 $\mu \mathrm{l}$ of $0.1 \mathrm{M}$ (pH 9.4) glycine buffer was pipetted into a cuvette, and $20 \mu \mathrm{l}(0.03$ units) of lyophilized uricase (Sigma Chemical Co.) in water was added. The cuvette was inverted to mix the components and $60-80 \mu \mathrm{l}$ of lithium carbonate extract of excretion products was added to the cuvette. The cuvette was inverted twice and the absorbance at $292 \mathrm{~nm}$ was recorded immediately and after 25 min. Preliminary studies demonstrated that all uric acid was converted to allantoin during this time. Unknown uric acid concentrations were estimated by comparing absorbances of samples with standard curves derived from samples of known uric acid concentration. Xanthine plus hypoxanthine were measured in a similar manner using the assay of Bergmeyer (1974).

\subsection{Feeding trials}

Feeding trials were performed to quantify the amount of food absorbed and converted to biomass by morphs of G. firmus. The trials were done on two diets to determine the effects of variation in nutrient quantity on nutrient absorption and conversion. All experiments were performed on unmated females since these females retain their eggs, and thus do not require access to oviposition material. This feature, together with other characteristics of unmated females described in Zera et al. (1998), greatly simplifies the measurement of nutritional indices. Importantly, mated and unmated Gryllus females exhibit very similar patterns of ovarian growth during the first week of adulthood (G. Cisper and A.J. Zera, unpublished data). Feeding trials were conducted over 7 days at $28^{\circ} \mathrm{C}$ under a 16L:8D photoperiod essentially as described previously (Zera et al., 1998) and were performed on females of the SEL-2 line [i.e. LW(f) and $L W(h)$ females of the block-2 line selected for the LW morph, and SW females of the block-2 line selected for the SW morph; L-2 and S-2 lines of Zera and Huang (1999)]. Briefly, on the day of adult molt (day 0), female crickets were weighed and placed in individual $16 \mathrm{oz}$. plastic cups. The crickets were fed either the standard $100 \%$ diet, or the $25 \%$ diet, in which the components of the $100 \%$ diet (excluding agar and mold inhibitor) were reduced to $25 \%$ of their original mass. The loss in mass was replaced by addition of an equivalent amount of $\alpha$ cel, non-nutritive cellulose, such that the $100 \%$ and $25 \%$ diets had the same dry mass. All juvenile crickets had been raised on the $100 \%$ diet during the last two stadia.

Weighed crickets were given a piece of preweighed food on day 0 . On days 2, 4, and 6, uneaten food was separated from the excretory products. Food and excreta were collected, placed in separate containers, and 
the crickets were given a fresh piece of food. Excretory products (feces and urine) were easily collected since they are produced together as semisolid (100\% diet) or solid ( $25 \%$ diet) pellets whose color differed from that of the food. On day 7, uneaten food and excreted matter were again collected and the crickets were weighed. Food and excreta were freeze-dried, weighed, and kept at $-80^{\circ} \mathrm{C}$ for later chemical analyses. The crickets were frozen for later dissection of flight muscles and ovaries. Initial (day 0) and final (day 7) dry masses of the G. firmus used in the feeding trial were estimated from their wet masses by linear regression of wet weights vs dry weights of separate groups of crickets raised under identical conditions. The dry mass of food consumed was estimated from the amount of wet diet consumed and standard curves of wet weight vs dry weight of diet obtained by linear regressions of each diet.

\subsection{Nutritional indices}

Absorption efficiency (AD, approximate digestibility), and efficiency of conversion of digested food into biomass (ECD) were calculated from the feeding trial data using standard formulae (Waldbauer, 1968; Woodring et al., 1979; van Loon, 1989). All calculations were done on dry masses of food, crickets and excreta. AD was estimated as the percentage of consumed food or specific macronutrient that was retained within the cricket during the 7-day feeding trial $\{[($ food consumed-material excreted $) /$ food consumed] $\times 100 \%$ \} ECD was the percentage of food retained by the cricket during the 7-day feeding trial that was converted into biomass $\{[$ (mass gain)/(food consumed-material excreted) $] \times 100 \%\}$.

Three types of AD and two types of ECD were calculated. First, uncorrected indices were estimated based on the dry masses of food consumed, excreta produced and mass gain of the cricket during the feeding trial. This is the standard method used to calculate $\mathrm{AD}$ and ECD in previous studies (Zera and Mole, 1994; Zera et al., 1998). Second, corrected AD and ECD values were calculated in the same manner, except that the total mass of excreted metabolites (uric acid, hypoxanthine, and xanthine) had been subtracted from the total mass of excreted material. Third, AD values for specific nutrients were calculated using the standard formula for AD given above, but using the masses of protein, carbohydrate or lipid in food and excreta.

\subsection{Organ masses}

Masses of ovaries and flight muscles (dorsolongitudinal and dorsoventral) were obtained as described previously (Mole and Zera, 1993; Zera et al., 1997).

\subsection{Statistical analyses}

Variation among morphs for excreted metabolites and corrected or uncorrected nutritional indices was analyzed by ANCOVA (Analysis of Covariance), essentially as described in Zera et al. (1998). The independent variable in the ANCOVA of a particular nutritional index was the numerator of that index, while the covariate was the denominator (Raubenheimer and Simpson, 1992; Zera et al., 1998). Similarly, the independent variable for ANCOVA of metabolite excretion was the total amount of that metabolite excreted during the 7-day feeding trial, and the covariate was the total dry mass of excreted material. Because of various covariatexdiet or line interactions, differences among individual morphs in absorption efficiency of carbohydrate, protein or lipid were analyzed for each line separately on each diet (see Section 2). In addition, a significant morph $\times$ covariate interaction, observed for carbohydrate $\mathrm{AD}$ for one of the control lines (CON-3), precluded analysis by ANCOVA. Data from this line were analyzed by the non-parametric Kruskal-Wallis test. A covariatexdiet interaction for AD for dry mass of diet was circumvented by analyzing the data from the $100 \%$ and $25 \%$ diets separately. No covariate $\times$ diet or morph interactions were observed for the ECD data, allowing analysis of the entire data set by ANCOVA.

Following the overall ANCOVA of AD (total dry mass of food) or ECD, three planned contrasts were performed to identify the specific source of variation among the morphs. These involved pairwise tests of each of the three morphs [LW(f) vs SW, LW(f) vs LW(h), and LW(h) vs SW]. Since these were planned contrasts, probabilities from these tests were not corrected for multiple comparisons. Finally, the amounts of excreted uric acid, hypoxanthine and xanthine were only estimated for a portion of crickets used in the feeding trials. Linear regressions of mass of excreted metabolite vs mass of excreta were undertaken so that metabolite excretion could be estimated for other crickets in these trials so that their corrected $\mathrm{ADs}$ and ECDs could be obtained.

\section{Results}

\subsection{Metabolite excretion}

The amount of uric acid or xanthine plus hypoxanthine per unit mass of excreta did not differ significantly among morphs within the control line (CON-3) or among selected morphs (SEL lines) on either diet (Table $1 ; P>0.15$ for each of the 12 ANCOVAs). Thus, there is no evidence to suggest that morphs in the unselected base population vary in metabolite excretion or that morphs vary genetically in metabolite excretion. In addition, values for individual morphs were typically very similar in control vs selected lines. 
Table 1. Mean ( \pm SEM) percentage of nitrogenous metabolites in excreta of wing and flight-muscle morphs of Gryllus firmus

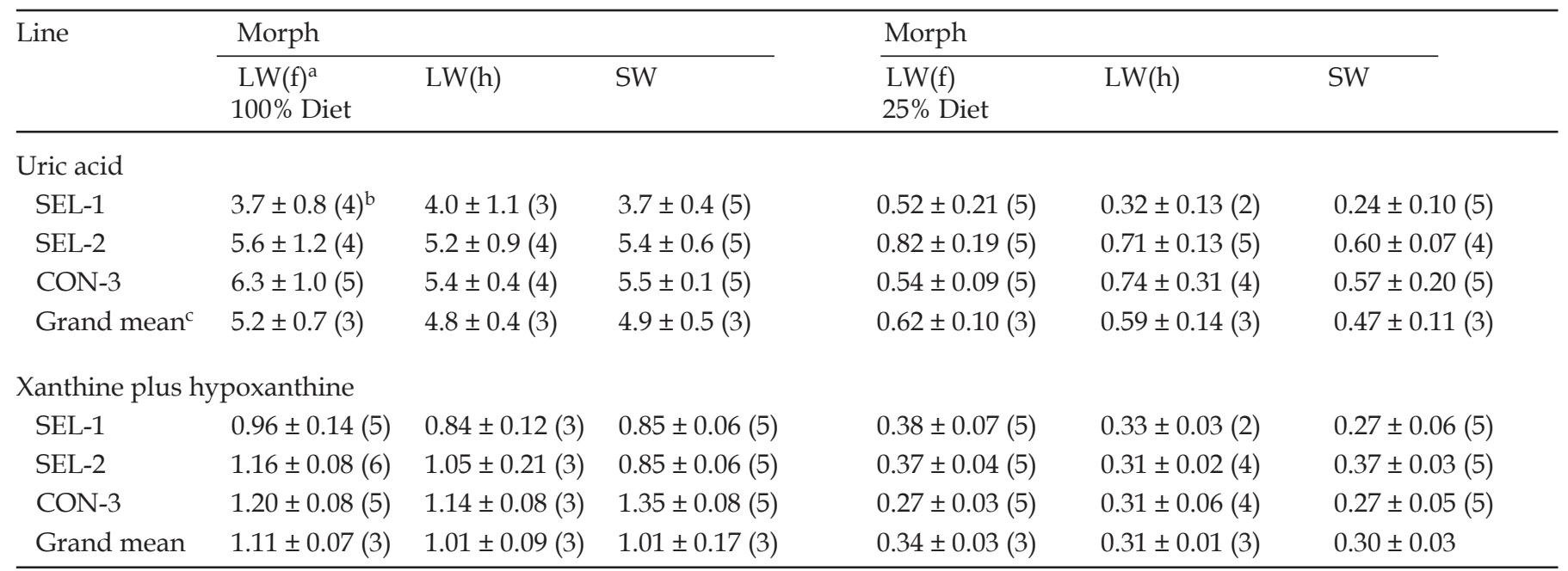

a $L W(f)=$ long wings with fully developed flight muscles; $L W(h)=$ long wings with histolyzed flight muscles; SW=short wings with underdeveloped flight muscles (see Section 2).

b Values are means \pm standard errors; sample sizes (number of crickets analyzed) are in parentheses.

c Mean of line means.

\subsection{Absorption efficiency $(A D)$ of macronutrients}

On the high diet, ADs were the highest for total soluble carbohydrate (86-93\%), followed by total lipid (73$79 \%$ ), and total protein (61-73\%) (Table 2, Table 3, and Table 4). The same rank order of ADs was observed on the low diet: carbohydrate AD (66-85\%), lipid AD (55$64 \%$ ), and protein $(23-54 \%)$. Indices were very similar
( $<5 \%$ difference) for different morphs within control or between selected lines, and for the same morph between control and selected lines (Table 2, Table 3, and Table 4). Grand mean ADs (morph means averaged over lines) for carbohydrate, lipid or protein were virtually identical for the three morphs fed a particular diet (Tables 2-4).

Table 2. Absorption efficiency (AD) of soluble carbohydrate for wing and flight muscle morphs of Gryllus firmus fed various diets

\begin{tabular}{|c|c|c|c|c|}
\hline \multirow[t]{2}{*}{ Line } & \multicolumn{3}{|l|}{ Morph } & \multirow[t]{2}{*}{ Results of ANCOVA or KW test } \\
\hline & $\operatorname{LW}(\mathrm{f})^{\mathrm{a}}$ & $\mathrm{LW}(\mathrm{h})$ & SW & \\
\hline \multicolumn{5}{|l|}{ 100\% Diet } \\
\hline SEL-2 & $91 \pm 0.8(10)$ & $87 \pm 0.7(9)$ & $87 \pm 0.4(10)$ & $* * *$ \\
\hline SEL-3 & $87 \pm 1.5(7)$ & $88 \pm 2.2(5)$ & $87 \pm 1.6(10)$ & NS \\
\hline Grand mean $^{\mathrm{d}}$ & $90 \pm 1.0(5)$ & $88 \pm 0.7(5)$ & $89 \pm 1.0(5)$ & NS \\
\hline \multicolumn{5}{|l|}{ 25\% Diet } \\
\hline SEL-1 & $85 \pm 0.7(9)$ & $85 \pm 1.4(2)$ & $82 \pm 0.6(10)$ & NS \\
\hline SEL-2 & $77 \pm 2.6(10)$ & $66 \pm 3.4(5)$ & $75 \pm 1.0(10)$ & $* * *$ \\
\hline Grand mean & $79 \pm 1.7(5)$ & $77 \pm 3.1(5)$ & $78 \pm 1.2(5)$ & NS \\
\hline
\end{tabular}

a $\mathrm{LW}(\mathrm{f})=$ long wings with fully developed flight muscles; $\mathrm{LW}(\mathrm{h})=$ long wings with histolyzed flight muscles; $\mathrm{SW}=$ short wings with underdeveloped flight muscles (see Section 2).

$\mathrm{b}$ Values are mean percentage \pm SEM; sample sizes are in parentheses; see Section 2 for calculation of AD.

c NS=non-significant $(P>0.1) ; * P<0.05 ; * * * P<0.005$.

$\mathrm{d}$ Average of line means. 
Table 3. Absorption efficiency (AD) of soluble protein for wing and flight muscle morphs of Gryllus firmus fed various diets

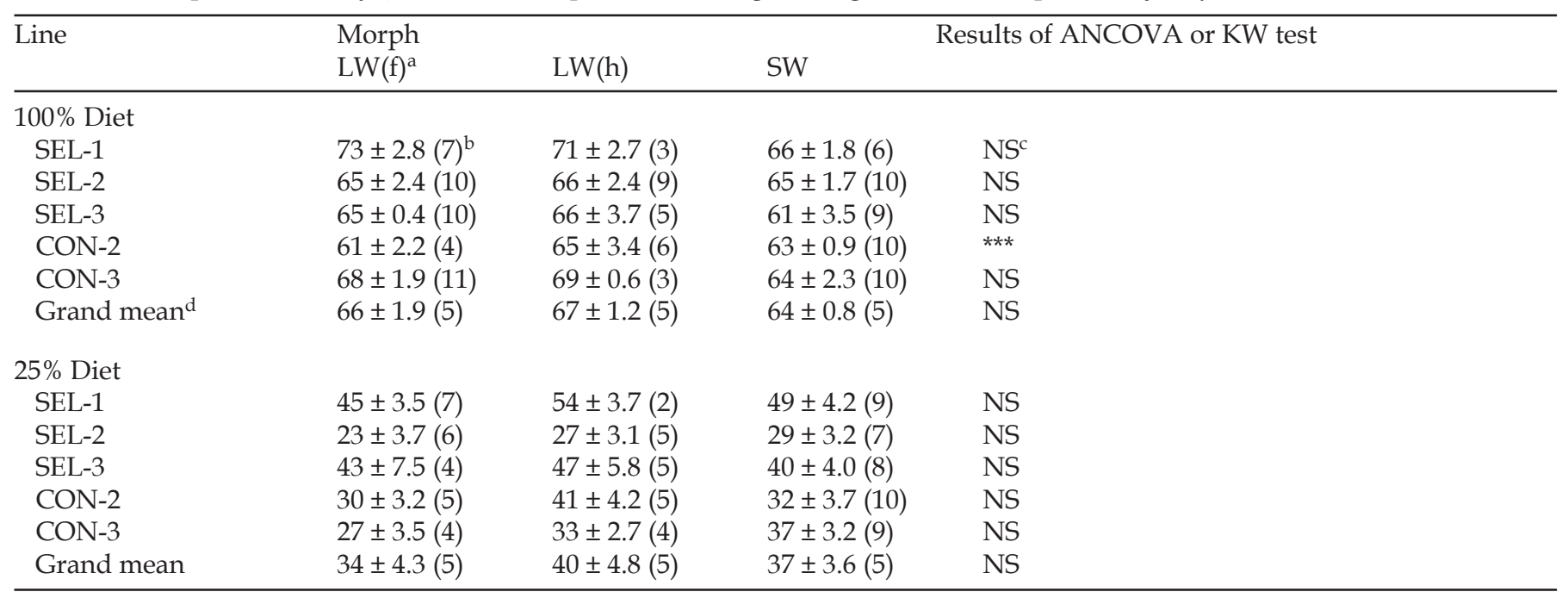

a $L W(f)=$ long wings with fully developed flight muscles; $L W(h)=$ long wings with histolyzed flight muscles; SW=short wings with underdeveloped

flight muscles (see Section 2).

b Values are mean percentage $\pm S E M$; sample sizes are in parentheses; see Section 2 for calculation of AD.

c NS = non-significant $(P>0.1) ; * * * 00.005$

$\mathrm{d}$ Average of line means.

Table 4. Absorption efficiency (AD) of total lipid for wing and flight muscle morphs of Gryllus firmus fed various diets

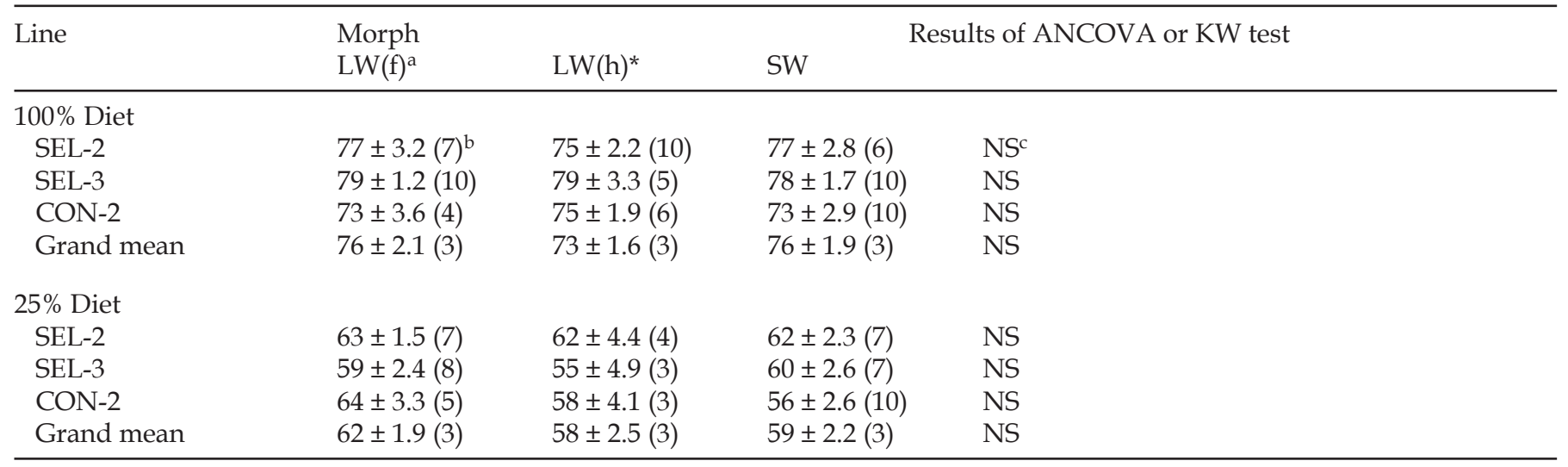

a $L W(f)=$ long wings with fully developed flight muscles; $L W(h)=$ long wings with histolyzed flight muscles; $S W=$ short wings with underdeveloped

flight muscles (see Section 2).

$\mathrm{b}$ Values are mean percentage \pm SEM; sample sizes are in parentheses; see Section 2 for calculation of AD.

c NS=non-significant $(P>0.1)$.

\subsection{Uncorrected and corrected $A D$ and $E C D$}

Uncorrected absorption efficiencies of total dry mass of food for the three morphs from one pair of selected lines (SEL-2) fed the high or low diets, and ADs corrected for metabolite excretion, are given in Table 5. An overall comparison of uncorrected vs corrected ADs could not be performed for the entire data set due to various covariatextreatment interactions. Hence corrected vs uncorrected values were compared separately for each morph on each diet. For the high diet, the corrected AD was significantly higher than the uncorrected $\mathrm{AD}$ for the
LW(f) morph $(P<0.025)$, the SW morph $(P<0.025)$ and was nearly significantly higher for the $\mathrm{LW}(\mathrm{h})$ morph $(P$ $=0.06)$. While statistically significant, differences between corrected and uncorrected values were small $(<$ $5 \%)$. For the low diet, none of the corrected ADs differed from the corresponding AD of the same morph $(P>0.25$ in each ANCOVA). The absence of differences on the low diet was likely due to the low concentration of excreted metabolites (see above).

Both corrected and uncorrected ADs exhibited essentially identical patterns of variation among the morphs (Table 5). Results of statistical analysis of corrected or 
Table 5. Corrected (C) and uncorrected (U) ECD and AD values for wing and flight muscle morphs of Gryllus firmus fed either the $100 \%$ or $25 \%$ diets

\begin{tabular}{|c|c|c|c|c|c|c|}
\hline \multirow[t]{3}{*}{ Diet/C or U } & \multicolumn{6}{|l|}{ Index } \\
\hline & \multicolumn{3}{|l|}{$\begin{array}{l}\text { ECD } \\
\text { Morph }\end{array}$} & \multicolumn{3}{|l|}{$\begin{array}{l}\mathrm{AD}^{\mathrm{a}} \\
\text { Morph }\end{array}$} \\
\hline & $\mathrm{LW}(\mathrm{f})^{\mathrm{b}}$ & LW(h) & SW & $\mathrm{LW}(\mathrm{f})$ & LW(h) & SW \\
\hline $100 \% \mathrm{C}$ & $31 \pm 1^{\mathrm{c}}$ & $41 \pm 2$ & $35 \pm 1$ & $68 \pm 1$ & $63 \pm 1$ & $64 \pm 1$ \\
\hline $100 \% \mathrm{U}$ & $32 \pm 1$ & $43 \pm 2$ & $36 \pm 1$ & $65 \pm 1$ & $60 \pm 1$ & $61 \pm 1$ \\
\hline $25 \%$ C & $-36 \pm 8$ & $12 \pm 4$ & $8 \pm 4$ & $23 \pm 2$ & $18 \pm 1$ & $18 \pm 1$ \\
\hline $25 \% \mathrm{U}$ & $-39 \pm 10$ & $13 \pm 5$ & $9 \pm 4$ & $22 \pm 2$ & $18 \pm 1$ & $17 \pm 1$ \\
\hline
\end{tabular}

a Absorption efficiency of total dry mass of diet.

$\mathrm{b}$ LW(f)=long wings with fully developed flight muscles; $\mathrm{LW}(\mathrm{h})=$ long wings with histolyzed flight muscles; SW=short wings with underdeveloped flight muscles (see Section 2).

c Values are mean percentage \pm standard error; sample sizes ranged from 36-38, 5-10 and 21-25, for LW(f), LW(h), and SW individuals, respectively; results of statistical analyses of these data are in Tables 6 and 7.

uncorrected values were essentially identical and only the results of analyses of corrected ADs will be presented here. Preliminary analyses of the ADs demonstrated a significant interaction between the covariate (total food consumed) and diet, thus precluding an overall ANCOVA of ADs on both diets. Hence, the ADs from each diet were analyzed separately. Corrected ADs differed significantly among the morphs fed either the high or low diets (Table 6). Pairwise comparisons of the three morphs indicated that all of the significant variation in $\mathrm{AD}$ was due to differences between the flight-capable, $\mathrm{LW}(\mathrm{f})$ morph, and either flightless morph [LW(h) or SW]. $L W(f)$ vs SW did not differ in AD on the high diet but differed on the low diet (Table 6). The AD of LW(f) differed from $\mathrm{LW}(\mathrm{h})$ on the high diet but not on the low

Table 6. Results of ANCOVAs of AD for wing and flight muscle morphs fed either a high nutrient $(100 \%)$ or low nutrient $(25 \%)$ diet

\begin{tabular}{|c|c|c|c|c|}
\hline \multirow{2}{*}{$\begin{array}{l}\text { Source of } \\
\text { variation } \\
\text { (degrees of } \\
\text { freedom) }\end{array}$} & \multicolumn{4}{|c|}{ Morphs compared } \\
\hline & All morphs & $\begin{array}{l}\text { LW(f) vs } \\
\text { SW }\end{array}$ & $\begin{array}{l}\mathrm{LW}(\mathrm{f}) \text { vs } \\
\mathrm{LW}(\mathrm{h})\end{array}$ & $\begin{array}{l}\text { LW(h) vs } \\
\text { SW }\end{array}$ \\
\hline \multicolumn{5}{|l|}{ 100\% Diet ${ }^{\mathrm{a}}$} \\
\hline Morph $(2,1)^{b}$ & $4.3^{* c, d}$ & 2.7 n.s. & $7.6^{* *}$ & 0.58 n.s. \\
\hline Covariate (1) & $280^{* * *}$ & $190^{* * *}$ & $31.4^{* * *}$ & $43.5^{* * *}$ \\
\hline Error & $63 d f$ & $54 d f$ & $40 d f$ & $28 d f$ \\
\hline \multicolumn{5}{|l|}{$25 \%$ Diet } \\
\hline Morph $(2,1)$ & $4.4^{*}$ & $8.2^{* *}$ & 2.8 n.s. & 0.09 n.s. \\
\hline Covariate (1) & $55.8^{* * *}$ & $52.5^{* * *}$ & $31.4^{* * *}$ & $96.4^{* * *}$ \\
\hline Error & $64 d f$ & $60 d f$ & $40 d f$ & $27 d f$ \\
\hline
\end{tabular}

a Because of significant Diet'Covariate interactions, ADs were analyzed separately on each diet by ANCOVA.

b Two df for tests of all (three) morphs, and one df for tests of two morphs.

c Values are F-ratios except for the Error term where values are degrees of freedom.

$\mathrm{d}^{* * *} P<0.005 ;{ }^{* *} P<0.01 ;{ }^{*} P<0.05 ;$ n.s. $=$ non-significant. diet (Table 6). Finally, LW(h) vs SW did not differ in AD on either diet (Table 4). The difference in AD between morphs was magnified on the low ( $25 \%$ difference) relative to the high diet $(<10 \%$ difference) (Table 5).

Corrected and uncorrected ECDs differed on average by $<7 \%$ (Table 5). In no case did corrected and uncorrected values differ significantly for a particular morph fed a particular diet. As was the case for AD, results of statistical analyses of ECD were identical for uncorrected or corrected values. Hence, only the results of analyses of corrected ECDs are presented. Preliminary analysis of ECDs showed no significant interactions between covariate and treatment. Therefore an overall ANCOVA (all three morphs and both diets) was performed. Significant effects due to both diet and morph were observed (Table 7), and no significant interaction was observed between these two factors (Table 7). Significant variation was due to an elevated ECD (1) in all morphs fed the high vs low diet and (2) in the flightless SW and LW(h) morphs vs the flight-capable LW(f) morph (Table 5 and Table 7).

Pairwise comparisons between morphs showed that ECD differed between LW(f) vs SW morphs over both diets and in each diet tested separately (Table 7). There was also a significant interaction between morph and diet. This interaction was caused by the substantially diminished (and negative) ECD of the LW(f) morph on the $25 \%$ diet, resulting in a much greater difference in ECD between the LW(f) and SW morphs on the low relative to the high diet (Table 5 and Table 7). ECD also was significantly lower in the LW(f) vs the LW(h) morph across both diets (Table 7$)$ and in each diet considered separately (high diet: $F_{(1,43)}=22.1, P<0.005$; low diet: $F_{(1,40)}$ $=11.5, P<0.005)$. Although ECDs also differed between the $\mathrm{LW}(\mathrm{f})$ and $\mathrm{LW}(\mathrm{h})$ morphs to a much greater degree on the $25 \%$ vs the $100 \%$ diets, no significant diet $\times$ morph interaction was observed (Table 7). This was likely due 
Table 7. Results of ANCOVAs of ECD for wing and flight muscle morphs fed either a high-nutrient $(100 \%)$ or lownutrient $(25 \%)$ diet

\begin{tabular}{|c|c|c|c|c|}
\hline \multirow{2}{*}{$\begin{array}{l}\text { Source of } \\
\text { variation } \\
\text { (degrees of } \\
\text { freedom) }\end{array}$} & \multicolumn{4}{|c|}{ Morphs compared } \\
\hline & $\begin{array}{l}\text { All } \\
\text { Morphs }\end{array}$ & $\begin{array}{l}\text { LW(f) vs } \\
\text { SW }\end{array}$ & $\begin{array}{l}\mathrm{LW}(\mathrm{f}) \text { vs } \\
\mathrm{LW}(\mathrm{h})\end{array}$ & $\begin{array}{l}\mathrm{LW}(\mathrm{h}) \mathrm{vs} \\
\text { SW }\end{array}$ \\
\hline $\operatorname{Morph}(2,1)^{\mathrm{a}}$ & $26.7^{* * * \mathrm{~b}, \mathrm{c}}$ & $32.7^{* * *}$ & $30.9^{* * *}$ & $6.5^{* * *}$ \\
\hline $\operatorname{Diet}(1)$ & $26.4^{* * *}$ & $33.6^{* * *}$ & $29.2^{* * *}$ & 1.6 n.s. \\
\hline $\mathrm{M}^{\prime} \mathrm{D}(2,1)$ & 2.7 n.s. & $4.1^{*}$ & 0.0 n.s. & 2.9 n.s. \\
\hline Covariate (1) & $53.6^{* * *}$ & $30.4^{* * *}$ & $32.9^{* * *}$ & $49.3^{* * *}$ \\
\hline \multirow[t]{3}{*}{ Error } & $131 d f$ & $115 d f$ & $86 d f$ & $58 d f$ \\
\hline & \multicolumn{4}{|c|}{$L W(f) v s W^{d}$} \\
\hline & $100 \%$ die & $25 \%$ diet & & \\
\hline Morph (1) & $4.4^{*}$ & $36.0^{* * *}$ & & \\
\hline Covariate (1) & $25.6^{* * *}$ & $14.3^{* * *}$ & & \\
\hline Error & $54 d f$ & $60 d f$ & & \\
\hline
\end{tabular}

a Two $d f$ for tests of all (three) morphs and one $d f$ for comparisons between pairs of morphs.

b Values are F-ratios except for the Error term, where values are degrees of freedom.

$\mathrm{C}^{* * * *} P<0.005 ;{ }^{*} P<0.05 ;$ n.s. $=$ non-significant $(P>0.1)$.

$\mathrm{d}$ Because of the significant M’D interaction, ECDs were also analyzed separately for each diet.

to the small sample size of $\mathrm{LW}(\mathrm{h})$ individuals in this study (see footnote of Table 5). Finally, ECDs also differed significantly between LW(h) and SW morphs (Table 5). However, ECDs were much more similar between these two flightless morphs than between either of these morphs and LW(f) when averaged over both diets [least-squared means $( \pm$ SEM) over both diets: $\mathrm{LW}(\mathrm{h})$ $=0.27 \pm 0.04, \mathrm{SW}=0.22 \pm 0.02, \mathrm{LW}(\mathrm{f})=-0.03 \pm 0.04]$ or on the low diet (Table 5 and Table 7). On the high diet, ECD for the SW morph was intermediate between the ECDs of the LW(f) and LW(h) morphs.

Mass of flight muscles and ovaries for the various morphs fed the $100 \%$ or $25 \%$ diets are given in Table 8 . Ovaries were small and similar in the morphs at adult emergence. However, ovaries were substantially greater in either flightless morph compared with the flight capable LW(f) morph by the end of the experiment (day 7). Moreover, the degree of elevation in ovarian growth in the flightless morph [either SW or LW(h)], relative to the flight-capable LW(f) morph, was magnified three-fold on the low nutrient (242-225\%) compared with the high nutrient (70-83\%) diet. Flight muscles were reduced in the flightless morph by $37-56 \%$. Flight muscle reduction was similar at all ages and on all diets.

\section{Discussion}

\subsection{Expression of morph-specific traits}

The degree of flight muscle reduction and enlargement of ovaries in flightless relative to flight-capable G. fir-
Table 8. Ovarian and flight muscle mass (as \% whole-body wet mass) for wing and flight-muscle morphs of Gryllus firmus fed the $100 \%$ or $25 \%$ diets $^{\mathrm{a}}$

\begin{tabular}{|c|c|c|c|}
\hline \multirow[t]{2}{*}{ Morph } & \multicolumn{3}{|l|}{ Organ mass } \\
\hline & Day-0 & $\begin{array}{l}\text { Day-7 }(100 \% \\
\text { Diet) }\end{array}$ & $\begin{array}{l}\text { Day-7 }(25 \% \\
\text { Diet) }\end{array}$ \\
\hline \multicolumn{4}{|l|}{ Ovaries } \\
\hline $\mathrm{LW}(\mathrm{f})$ & $2.0 \pm 0.2(n=13)^{\mathrm{b}}$ & $14.3 \pm 0.7(n=36)$ & $3.1 \pm 0.2(n=38)$ \\
\hline LW(h) & - & $\begin{array}{l}26.1 \pm 0.8(n=10) \\
(83 \%)^{c}\end{array}$ & $\begin{array}{l}10.1 \pm 0.6(n=5) \\
(225 \%)\end{array}$ \\
\hline SW & $\begin{array}{l}2.3 \pm 0.7(n=7) \\
(15 \%)\end{array}$ & $\begin{array}{l}24.3 \pm 1.3(n=22) \\
(70 \%)\end{array}$ & $\begin{array}{l}10.6 \pm 0.8(n=25) \\
(242 \%)\end{array}$ \\
\hline \multicolumn{4}{|c|}{ Flight muscles } \\
\hline $\mathrm{LW}(\mathrm{f})$ & $(n=13)$ & $12.3 \pm 0.2(n=36)$ & $13.8 \pm 0.2(n=38)$ \\
\hline LW(h) & - & $\begin{array}{l}7.8 \pm 0.2(n=10) \\
(37 \%)^{\mathrm{d}}\end{array}$ & $\begin{array}{l}8.5 \pm 0.3(n=5) \\
(38 \%)\end{array}$ \\
\hline SW & $\begin{array}{l}5.5 \pm 0.4(n=7) \\
(56 \%)\end{array}$ & $\begin{array}{l}6.0 \pm 0.2(n=22) \\
(51 \%)\end{array}$ & $\begin{array}{l}6.5 \pm 0.2(n=25) \\
(52 \%)\end{array}$ \\
\hline \multicolumn{4}{|c|}{$\begin{array}{l}\text { a Crickets were fed either the } 100 \% \text { or } 25 \% \text { diet starting on day } 0 \text { of } \\
\text { adulthood. Prior to this time all crickets had been fed the } 100 \% \\
\text { diet. }\end{array}$} \\
\hline \multicolumn{4}{|c|}{$\begin{array}{l}\text { b Mean ( } \pm \text { SEM) percent ovarian or flight-muscle mass ( } n=\text { sample } \\
\text { size). }\end{array}$} \\
\hline \multicolumn{4}{|c|}{$\begin{array}{l}\text { c Percent increase in ovarian mass relative to } \mathrm{LW}(\mathrm{f}) \\
\mathrm{d} \text { Percent decrease in flight-muscle mass relative to } \mathrm{LW}(\mathrm{f})\end{array}$} \\
\hline
\end{tabular}

mus, observed in the present study (Table 8), is similar to data reported in previous studies of naturally-occurring or hormonally-induced dispersal polymorphism (Tanaka, 1993; Mole and Zera, 1993; Zera et al., 1997; Zera and Denno, 1997). These results indicate that nutrient absorption and utilization were measured under experimental conditions in which the typical trade-off between flight capability and early reproduction is seen.

\subsection{Efficiency of absorption of macronutrients by morphs}

Nutrient absorption (AD) by wing and flight muscle morphs of G. firmus is relevant to two key trade-off issues. The first concerns the physiological processes responsible for functionally-important variation in chemical constituents between adult morphs. For example, the flight-capable morph of two Gryllus species has higher triglyceride/total lipid content than the flightless morph (Zera et al., 1994, 1998), which appears to be an important adaptation for flight (Zera et al., 1999). However, the physiological processes responsible for these lipid differences are unknown. The key issue is whether variation among morphs arises from differential absorption of lipid from the diet (no trade-off), or from differential allocation of assimilated nutrients to lipid biosynthesis or catabolism (trade-off).

In the present study, no biologically-significant phenotypic or genetic differences were observed between adult morphs in assimilation of total lipid, carbohydrate, or protein (Table 2, Table 3, and Table 4). Hence, variation in chemical composition between wing morphs, described above, appears to result from variation in some 
process other than absorption of specific macronutrients per unit mass of food consumed by adults. We are currently investigating the potential role of differential lipid and carbohydrate biosynthesis or catabolism in producing morph-specific differences in the concentration of these macromolecules. Carbohydrate and protein ADs for G. firmus fed the $100 \%$ diet were similar to values for two other orthopterans: adult female Acheta domesticus fed an artificial diet (Woodring et al., 1979) and last stadium Locusta migratoria fed wheat seedlings (Simpson, 1982). Total lipid ADs for G. firmus also were similar to values for adult female $A$. domesticus and were higher than values for juvenile L. migratoria (Woodring et al., 1979; Simpson, 1982).

Data on morph-specific absorption of specific macronutrients are also important in assessing the significance of variation in $\mathrm{AD}$ among morphs for total dry mass of food. Previous studies have shown that wing or flight muscle morphs absorb an equivalent proportion of consumed food (G. rubens, Mole and Zera, 1993; G. assimilis, Zera et al., 1998), or that the long-winged morph absorbs a slightly (14\%) greater proportion of dry mass of consumed food (G. firmus; Zera and Mole, 1994; present study). On the other hand, conversion of assimilated nutrients to biomass (ECD) was elevated by $45-100 \%$ in the flightless vs flight-capable or LW morph in each of these species [LW(f) and LW(h) morphs were not distinguished in previous studies of G. rubens and G. firmus]. Hence, increased nutrient conversion was viewed as being more important than increased nutrient absorption in providing additional nutrients for enhanced egg production of flightless females. This argument is based on the assumption that adult morphs do not differentially absorb nutrients from the diet and hence the relative amount of dry food absorbed is a measure of the relative amount of calories absorbed by each morph. Results of the present study support this assumption. Despite the voluminous literature on insect nutritional ecology (Waldbauer, 1968; Woodring et al., 1979; Simpson, 1982; Slansky and Scriber, 1985; van Loon, 1989; Bernays, 1989; Simpson and Simpson, 1989), the present and earlier studies of Gryllus morphs represent the first studies of polymorphic and genetic variation for nutritional indices.

\subsection{Metabolite excretion and nutritional indices}

Studies of metabolism and energetics of organisms such as insects (and birds) are complicated by the excretion of feces and urine together. Measurement of absorption efficiency requires that the mass of end products of metabolism be distinguished from unabsorbed food in the combined feces and urine (Waldbauer, 1968; Slansky and Scriber, 1985; van Loon, 1989). The present study focused on the excretion of uric acid, hypoxanthine and xanthine by morphs of G. firmus. Uric acid is the end product of nitrogen metabolism that is most commonly excreted by insects as a group and by the cricket, $A$. domesticus (Bursell, 1967; Cochran, 1985). Recent studies also have shown that xanthine and hypoxanthine can account for a significant amount of total purine excreted by some species (Bursell, 1967; Bhattacharya and Waldbauer, 1972; Cockran, 1985). Percentages of uric acid, hypoxanthine and xanthine in dry mass of excreta of G. firmus fed the $100 \%$ or $25 \%$ diets (Table 1 ) were roughly similar to values for other insects fed artificial diets (Waldbauer, 1968; Bhattacharya and Waldbauer, 1972; Woodring et al. 1979).

End products of nitrogen metabolism comprised only a small proportion of the dry mass of excreta $(<8 \%$ for the $100 \%$ diet and $<2 \%$ for the $25 \%$ diet). The concentration of these end products did not differ between morphs, had only a minor effect on the absolute values of AD or ECD for a particular morph, and had no significant influence on the pattern of variation among morphs for these indices (Table 5; Section 3). Thus, conclusions drawn from previous comparisons of nutritional indices among morphs (Zera and Denno, 1997; Zera et al., 1998) were likely unaffected by the fact that indices were not corrected for excretion of nitrogenous metabolites.

\subsection{Morph-specific variation in nutrient absorption and utilization}

Identifying the physiological causes underlying the trade-off between ovarian growth and flight capability requires detailed information on relative nutrient consumption, absorption and allocation by wing and flightmuscle morphs (Mole and Zera, 1993; Zera and Denno, 1997; Zera et al., 1998). ECD is a nutritional index that has been used extensively in studies of insect feeding (Waldbauer, 1968; Slansky and Scriber, 1985; van Loon, 1989; and Woodring et al., 1979), and in trade-off studies in species of Gryllus (reviewed in Zera and Denno, 1997; Zera et al., 1998). ECD measures the proportional allocation of absorbed resources to whole-organism biomass (gain in mass of both somatic and reproductive tissues) vs whole-organism respiration (Waldbauer, 1968; Woodring et al., 1979; Slansky and Scriber, 1985). For example, the corrected ECD of $31 \pm 1 \%$ for LW(f) females fed the $100 \%$ diet (Table 5) means that, during the first week of adulthood, $31 \%$ of the dry mass of nutrients assimilated from the diet were converted into whole organism somatic and reproductive tissue, while $69 \%$ were utilized for respiration (lost from the organism as $\mathrm{CO}_{2}$ ). Significant variation in ECD between morphs documents the differential allocation (trade-off) of nutrients to whole-organism biomass vs respiration. Thus, the higher ECD in flightless vs flight-capable morphs in each of three Gryllus species (Mole and Zera, 1993; Zera and Denno, 1997; Zera et al., 1998) indicates 
that a greater proportion of assimilated nutrients are allocated to biomass gain vs respiration in the flightless morph of each of these species. A large proportion of biomass gain $(>75 \%)$ in adult Gryllus species and in the phylogenetically related $A$. domesticus is due to ovarian growth (Woodring et al., 1979; Zera et al., 1998; A. Zera, unpublished data). Thus elevated ECD in the flightless morph, to a large extent, reflects the increased allocation of nutrients to ovarian growth vs whole organism respiration (Zera and Denno, 1997; Zera et al., 1998). That is, ovarian growth trades-off with whole-organism respiration.

Previous estimates of ECD, AD and other nutritional indices (e.g. consumption and growth) in naturally-occurring wing morphs (Mole and Zera, 1993, 1994) had several limitations. First, since only a single diet $(100 \%$ diet) was used in these studies, the extent to which observed nutritional index variation between morphs was dependent upon that diet was unknown. In addition, LW morphs with functional vs histolyzed flight muscles $[L W(f)$ vs $L W(h)]$ were not distinguished. This potentially masked physiological differences between flightless and flight-capable individuals because the LW "flight-capable" group was a mixture of individuals some of which were flightless.

In the present study, nutritional indices were estimated for each of three wing- and flight-muscle morphs that were raised on diets of high or low nutrient quantities. An important finding was that ECD was significantly elevated in each type of flightless morph [LW(h) or SW] relative to the long-winged, flight-capable morph [LW(f)] over all diets, and on each diet considered separately (Section 3, Table 5 and Table 7). Similarly, AD was moderately reduced in each flightless morph, relative to the flight-capable morph, on either the $25 \%$ or $100 \%$ diet (Table 5). These results corroborate our previous findings on morph-specific variation in nutritional indices in G. firmus discussed above. Results also show that flightless morphs that are produced by modification of different developmental pathways (blockage of muscle development vs histolysis of developed muscles) during different times of the life cycle (juveniles vs adults), in general, assimilate and convert nutrients in a similar manner. These results strengthen our previous conclusions that physiological and reproductive features are more similar between flight muscle morphs that differ in wing length [LW(h) and SW] than between wing length morphs that differ in flight muscles [LW(f) and LW(h)] (Zera et al., 1997, 1999).

Another important finding of the present study was that ECD was elevated in flightless vs flight-capable morphs to a much greater degree on the low nutrient (25\%) compared with the high nutrient $(100 \%)$ diet (Table 5 and Table 7). Moreover, ECD was negative for the LW(f) morph on the $25 \%$ diet (Table 5), indicating that nutrient intake was not sufficient to meet the energetic requirements of maintenance metabolism and activity. Thus the LW(f) morph used body reserves to fuel respiration and lost weight on the $25 \%$ diet during the experiment. A similar magnification of ECD between (a hormonally-induced) flightless and flight-capable morphs was observed on a low nutrient diet in the congener, G. assimilis (Zera et al., 1998). In both the study of Zera et al. (1998) and the present study, the greater magnification of ECD between morphs on the low nutrient diet was associated with a greater magnification of ovarian growth (Table 8 ). These studies clearly show that tradeoffs of internal resources and resulting fecundity variation can be strongly affected by nutrient availability.

The validity of our conclusions on trade-offs in G. firmus and other Gryllus species is contingent upon the reliability of nutritional indices as indicators of nutrient allocation to respiration. Recently, Tanaka and Suzuki (1998, p. 128) have questioned the use of nutritional indices for this purpose, claiming that these indices only provide an indirect measure of respiration and are inferior to direct measures of oxygen $\left(\mathrm{O}_{2}\right)$ consumption. This claim is based on a misunderstanding of the bioenergetic definition of respiration, and the relationship between $\mathrm{O}_{2}$ consumption and respiration. In bioenergetics, respiration is defined as the amount of calories devoted to metabolism that is lost from the organism (i.e. respiratory metabolism), and is not the quantity of $\mathrm{O}_{2}$ consumed or $\mathrm{CO}_{2}$ expired (Withers, 1992, pp. 83-88). Both respirometry (e.g. quantifying $\mathrm{O}_{2}$ consumption) and nutritional indices are indirect measures of respiration. Estimation of respiratory metabolism from the energy contents or masses of food consumed and material excreted is considered preferable by many to estimates based on respirometry, and the estimation of respiration from dry mass or caloric content of food assimilation is a standard practice in energetics (Muthukrishnan and Pandain, 1987; Wrightman, 1981). Ironically, closed-vessel respirometry, the method used by Tanka and co-workers (e.g. Tanaka and Okuda, 1996), is especially prone to experimental artifacts ("flask effects"; Wightman, 1981; McEvoy, 1985; Muthukrishnan and Pandain, 1987), which is why we did not use this method. We emphasize that we have thus far quantified the trade-off between respiration and ovarian growth in terms of dry mass of assimilated food. We are currently estimating energy budgets for each morph to quantify the tradeoff in units of energy. Our preliminary results indicate that the pattern of trade-offs between the morphs is very similar whether determined gravimetrically (Table 5) or in units of energy.

\subsection{The importance of absorption efficiency $(A D)$ in trade-off studies}

Nutrient absorption efficiency has long been viewed as an essential component of energetics studies (Muth- 
ukrishnan and Pandian, 1987; Withers, 1992). By contrast, the importance of this measure in life history tradeoff studies has been much less appreciated, and this index often has not been quantified. However, phenotypic differences in the allocation of internal nutrients to various organismal features (i.e. a nutrient-based tradeoff) can only be rigorously documented if the quantity of absorbed nutrients is quantified for the phenotypes whose allocation patterns are being compared (Mole and Zera, 1993; Zera and Denno, 1997). Thus, claims of internal trade-offs in studies where absorption efficiency has not been estimated (e.g. Djawdan et al., 1996; some experiments in Tanaka, 1993) should be viewed with caution. As an alternate approach, Tanka and co-workers (Tanaka, 1993; and Tanaka and Suzuki, 1998) have attempted to eliminate nutrient assimilation from consideration by conducting trade-off studies on starved insects. However, this approach limits trade-off studies to a single highly restrictive condition: total starvation. By contrast, a nutritional index approach allows nutrient allocation to be scaled to amount of food absorbed. Hence trade-offs of internal resources can be quantified under any degree of nutrient intake. This allows tradeoffs to be quantified under a range of nutrient conditions that might be encountered by the organism in the field. Moreover, this approach allows the investigator to quantify the relative importance of consumption, assimilation and allocation to some organismal feature (e.g. increased ovarian growth in the flightless morph). Alteration of nutrient intake is an exceedingly important adaptation of insects for dealing with variation in nutrient quantity and quality (Simpson and Simpson, 1989; Zanotto et al., 1993). Variation in nutrient intake can strongly influence the magnitude of internal tradeoffs (Table 5 and Table 8; Zera and Denno, 1997; Zera et al., 1998). Thus, any comprehensive study of a trade-off should investigate how variation in absorption or consumption influences allocation. While nutritional indices certainly do not provide all the necessary information on internal trade-offs (Mole and Zera, 1993), they are indispensable tools in such studies.

\section{Acknowledgments}

This study was supported by grants IBN 9507388 and IBN 9808249 from the National Science Foundation to AJZ. T. Brink was supported by funds from a Howard Hughes Grant to the School of Biological Sciences, University of Nebraska and by a Research Experience for Undergraduates (REU) supplement to the NSF grant IBN-9507388. Data reported in this study are from an Honors Thesis submitted by T. Brink to the University of Nebraska as partial fulfillment for a Bachelor of Science Degree with Highest Distinction. We thank T. Rooneem for help in the chemical analyses.

\section{References}

Bergmeyer, H.L., 1974. Methods of Enzymatic Analysis, vol. 1, Academic Press, New York.

Bernays, E.A., Editor, , 1989. Insect-Plant Interactions, vol. II, CRC Press, Boca Raton, FL.

Bhattacharya, A.K. and Waldbauer, G.P., 1969. Quantitative determination of uric acid in insect feces by lithium carbonate extraction and the enzymatic spectrophotometric method. Annals of the Entomological Society of America 62, pp. 925-927.

Bhattacharya, A.K. and Waldbauer, G.P., 1972. The effect of diet on the nitrogenous end products excreted by larval Tribolium confusum: with notes on correction of A.D. and E.C.D. for fecal urine. Entomologia Experimentalis et Applicata 15, pp. 238-247.

Bursell, E., 1967. The excretion of nitrogen in insects. Advances in Insect Physiology 4, pp. 33-67.

Cochran, D.G., 1985. Nitrogenous excretion. In: Kerkut, G.A. and Gilbert, L.I., Editors, 1985. Comprehensive Insect Biochemistry, Physiology and Pharmacology, vol. 4, Pergamon Press, Oxford, pp. 467-506.

Dingle, H., 1996. Migration: the Biology of Life on the Move, Oxford University Press, Oxford.

Djawdan, M., Sugiyama, T.T., Schlaeger, L.K., Bradley, T.J. and Rose, M.R., 1996. Metabolic aspects of the trade-off between fecundity an longevity in Drosophila melanogaster. Physiological Zoology 69, pp. 1176-1195.

Hardie, J. and Lees, A.D., 1985. Endocrine control of polymorphism and polyphenism. In: Kerkut, G.A. and Gilbert, L.I., Editors, 1985. Comprehensive Insect Biochemistry, Physiology and Pharmacology, vol. 8, Pergamon Press, Oxford, pp. 441-490.

Harrison, R.G., 1980. Dispersal polymorphisms in insects. Annual Review of Ecology and Systematics 11, pp. 95-118.

McEvoy, P.B., 1985. Balancing insect energy budgets. Oecologia 66, pp. $154-156$.

Mole, S. and Zera, A.J., 1993. Differential allocation of resources underlies the dispersal-reproduction trade-off in the wing-dimorphic cricket Gryllus rubens. Oecologia 93, pp. 121-127.

Mole, S. and Zera, A.J., 1994. Differential resource consumption obviates a potential flight-fecundity trade-off in the sand cricket (Gryllus firmus). Functional Ecology 8, pp. 573-580.

Muthukrishnan, J. and Pandian, T.J., 1987. Insecta. In: Pandian, T.J. and Vernberg, F.J., Editors, 1987. Animal Energetics, vol. 1, Academic Press, San Diego, CA, pp. 373-511.

Nijhout, H.F., 1999. Control mechanisms of polyphenic development in insects. Bioscience 49, pp. 181-192.

Raubenheimer, D. and Simpson, S.J., 1992. Analysis of covariance: an alternate to nutritional indices. Entomologia Experimentalis et Applicata 62, pp. 221-231.

Roff, D.A., 1986. The evolution of wing dimorphism in insects. Evolution 40, pp. 1009-1020.

Simpson, S.J., 1982. Changes in the efficiency of utilization of food throughout the fifth-instar nymphs of Locusta migratoria. Entomologia Experimentalis et Applicata 31, pp. 265-275. 
Simpson, S.J. and Simpson, C.L., 1989. The mechanisms of nutritional compensation by phytophagous insects. In: Bernays, E.A., Editor, , 1989. Insect-Plant Interactions, vol. II, CRC Press, Boca Raton, FL, pp. 111-160.

Slansky, F.J. and Scriber, J.M., 1985. Food consumption and utilization. In: Kerkut, G.A. and Gilbert, L.I., Editors, 1985. Comprehensive Insect Biochemistry, Physiology and Pharmacology, vol. 4, Pergamon Press, Oxford, pp. 87-163.

Stoschek, C.M., 1990. Quantitation of protein. Methods in Enzymology 182, pp. 50-68.

Tanaka, S., 1993. Allocation of resources to egg production and flight muscle development in a wing dimorphic cricket Modicogryllus confirmatus. Journal of Insect Physiology 39, pp. 493-498.

Tanaka, S. and Okuda, T., 1996. Effects of photoperiod on sexual maturation, fat content and respiration rate in adult Locusta migratoria. Japanese Journal of Entomology 64, pp. 420-428.

Tanaka, S. and Suzuki, Y., 1998. Physiological trade-off between reproduction, flight capability and longevity in a wing-dimorphic cricket Modicogryllus confirmatus. Journal of Insect Physiology 44, pp. 121-129.

Van Handel, E., 1985. Rapid determination of glycogen and sugars in mosquitoes. Journal of the American Mosquito Control Association 1, pp. 299-301.

van Loon, J.J.A., 1989. Measuring food utilization in plantfeeding insects - toward a metabolic dynamic approach. In: Bernays, E.A., Editor, , 1989. Insect-Plant Interactions, vol. 3, CRC Press, Boca Raton, FL, pp. 79-124.

Veazy, J.N., Kay, C.A.R., Walker, T.J. and Whitcomb, W.H., 1976. Seasonal abundance, sex ratio, and macroptery of field crickets in northern Florida. Annals of the Entomological Society of America 69, pp. 374-380.

Waldbauer, G.P., 1968. The consumption and utilization of food by insects. Advances in Insect Physiology 5, pp. 229-288.

Wightman, J.A., 1981. Why insect energy budgets do not balance. Oecologia 50, pp. 166-169.
Withers, P.C., 1992. Comparative Animal Physiology, Saunders College, Forth Worth, TX.

Woodring, J.P., Clifford, C.W. and Beckman, B.R., 1979. Food utilization and metabolic efficiency in larval and adult house crickets. Journal of Insect Physiology 25, pp. 903-912.

Zanotto, E.P., Simpson, S.J. and Raubenheimer, D., 1993. The regulation of growth by locusts through post-ingestive compensation for variation in the levels of dietary protein and carbohydrate. Physiological Entomology 18, pp. $425-434$

Zera, A.J. and Denno, R.F., 1997. Physiology and ecology of dispersal polymorphism in insects. Annual Review of Entomology 42, pp. 207-231.

Zera, A.J. and Huang, Y., 1999. Evolutionary endocrinology of juvenile hormone esterase: functional relationship with wing polymorphism in the cricket Gryllus firmus. Evolution 53, pp. 837-847.

Zera, A.J. and Mole, S., 1994. The physiological costs of flight capability in wing-dimorphic crickets. Researches on Population Ecology (Kyoto) 36, pp. 151-156.

Zera, A.J., Mole, S. and Rokke, K., 1994. Lipid, carbohydrate and nitrogen content of long- and short-winged Gryllus firmus: implications for the physiological cost of flight capability. Journal of Insect Physiology 40, pp. 1037-1044.

Zera, A.J., Sall, J. and Grudzinski, K., 1997. Flight-muscle polymorphism in the cricket Gryllus firmus: muscle characteristics and their influence on the evolution of flightlessness. Physiological Zoology 70, pp. 519-529.

Zera, A.J., Potts, J. and Kobus, K., 1998. The physiology of life history trade-offs: experimental analysis of a hormonallyinduced life history trade-off in Gryllus assimilis. American Naturalist 152, pp. 7-23.

Zera, A.J., Sall, J. and Otto, K., 1999. Biochemical aspects of flight and flightlessness in Gryllus: flight fuels, enzyme activities and electrophoretic profiles of flight muscles from flight-capable and flightless morphs. Journal of Insect Physiology 45, pp. 275-286. 